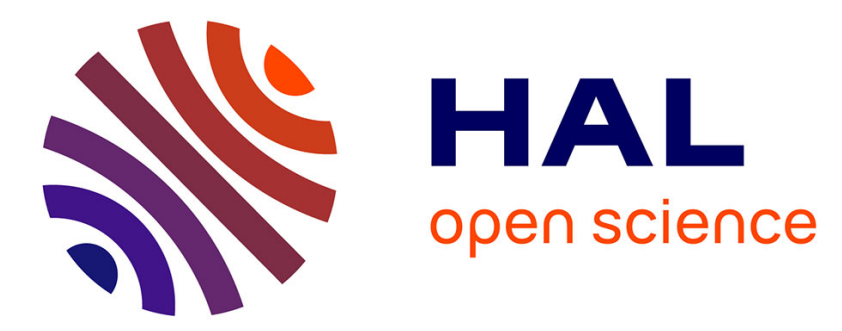

\title{
Study of the production of strange and multi-strange particles in lead-lead interactions at the CERN SPS: the NA57 experiment
}

\author{
F. Antinori, A. Badala, R. Barbera, I.J. Bllodworth, M. Botje, R. Caliandro, \\ M. Campbell, E. Cantatore, W. Carena, N. Carrer, et al.
}

\section{To cite this version:}

F. Antinori, A. Badala, R. Barbera, I.J. Bllodworth, M. Botje, et al.. Study of the production of strange and multi-strange particles in lead-lead interactions at the CERN SPS: the NA57 experiment. Nuclear Physics A, 2001, 681, pp.165-173. in2p3-00019671

\section{HAL Id: in2p3-00019671 https://hal.in2p3.fr/in2p3-00019671}

Submitted on 5 Sep 2001

HAL is a multi-disciplinary open access archive for the deposit and dissemination of scientific research documents, whether they are published or not. The documents may come from teaching and research institutions in France or abroad, or from public or private research centers.
L'archive ouverte pluridisciplinaire HAL, est destinée au dépôt et à la diffusion de documents scientifiques de niveau recherche, publiés ou non, émanant des établissements d'enseignement et de recherche français ou étrangers, des laboratoires publics ou privés. 


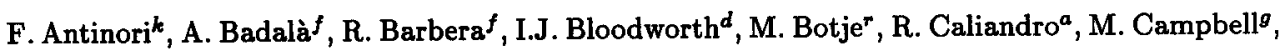
E. Cantatore ${ }^{g}$, W. Carena ${ }^{g}$, N. Carrer ${ }^{k}$, A.P. de Haas ${ }^{r}$, D. Di Bari ${ }^{a}$, S. Di Liberto ${ }^{n}$, R. Divia ${ }^{g}$, D. Elia ${ }^{a}$, D. Evans ${ }^{d}$, K. Fanebust ${ }^{b}$, J. Fedorisin ${ }^{h}$, G.A. Feofilov ${ }^{p}$, R.A. Fini ${ }^{a}$, J. Ftáčnik ${ }^{e}$, B. Ghidinia , G. Grella ${ }^{o}$, M. Gulino ${ }^{f}$, H. Helstrup ${ }^{c}$, A.K. Holme ${ }^{j}$, A. Jacholkowski ${ }^{a}$, G.T. Jones ${ }^{d}$, P. Jovanovic $^{e}$, A. Jusko ${ }^{h}$, R. Kamermans ${ }^{r}$, J.B. Kinson ${ }^{d}$, W. Klempt ${ }^{g}$, K. Knudson ${ }^{g}$, B. Kocper ${ }^{h}$, A.A. Kolojvari ${ }^{p}$, I. Králik ${ }^{h}$, P. Kuijer ${ }^{r}$, V. Lenti ${ }^{a}$, R. Lietava ${ }^{e}$, G. Løvhøiden ${ }^{j}$, M. Lupták ${ }^{h}$, V. Manzari ${ }^{a}$, M.A. Mazzoni ${ }^{n}$, G. Martinska ${ }^{h}$, F. Meddi ${ }^{n}$, A. Michalon ${ }^{q}$, M.E. Michalon-Mentzer ${ }^{q}$, M. Morando ${ }^{k}$, D. Muigg ${ }^{r}$, E. Nappi ${ }^{a}$, F. Navach ${ }^{a}$, P.I. Norman ${ }^{d}$, A. Palmeri ${ }^{f}$, G.S. Pappalardo ${ }^{f}$,

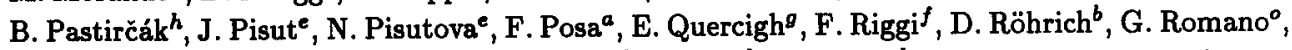
K. Šafařík ${ }^{g}$, L. Śándor ${ }^{h}$, E. Schillings ${ }^{r}$, M. Senè ${ }^{l}$, R. Senè ${ }^{l}$, G. Segato ${ }^{k}$, W. Snoeys ${ }^{g}$, P. Staroba $^{m}$, M. Thompson ${ }^{d}$, G. Tomasicchio ${ }^{a}$, G.D. Torrieri ${ }^{d}$, T.A. Tulina ${ }^{p}$, T.S. Tveter ${ }^{j}$, J. Urbán $^{h}$, F. Valiev ${ }^{p}$, A. van den Brink ${ }^{r}$, P. van de Ven ${ }^{r}$, P. Vande Vyvre ${ }^{g}$, N. van Eijndhoven, L. Vannucci ${ }^{i}$, A. Vascotto ${ }^{g}$, O. Villalobos Baillie ${ }^{d}$, I. Vinogradov ${ }^{p}$, T. Virgili ${ }^{o}$, M.F. Votruba ${ }^{d}, J$. Vrlakova ${ }^{h}$ and P. Závada ${ }^{m}$

a Dipartimento IA di Fisica dell'Università e del Politecnico di Bari and INFN, Bari, Italy

${ }^{b}$ Fysisk Institutt, Universitetet i Bergen, Bergen, Norway

${ }^{c}$ Høgskolen i Bergen, Bergen, Norway

d University of Birmingham, Birmingham, UK

e Comenius University, Bratislava, Slovakia

${ }^{f}$ University of Catania and INFN, Catania, Italy

g CERN, European Laboratory for Particle Physics, Geneva, Switzerland

${ }^{h}$ Institute of Experimental Physics SAS and Safárik University, Kosice, Slovakia

i INFN, Laboratori Nazionali di Legnaro, Legnaro, Italy

${ }^{j}$ University of Oslo, Oslo, Norway

${ }^{k}$ University of Padua and INFN, Padua, Italy

${ }^{l}$ Collège de France and IN2P3, Paris, France

${ }^{m}$ Institute of Physics, Prague, Czech Republic

${ }^{n}$ University "La Sapienza" and INFN, Rome, Italy

o Dipartimento di Scienze Fisiche "E.R. Caianiello" dell'Università and INFN, Salerno, Italy

${ }^{p}$ State University of St. Petersburg, St. Petersburg, Russia

${ }^{q}$ IReS/ULP, Strasbourg, France

r Utrecht University and NIKHEF, Utrecht, The Netherlands 

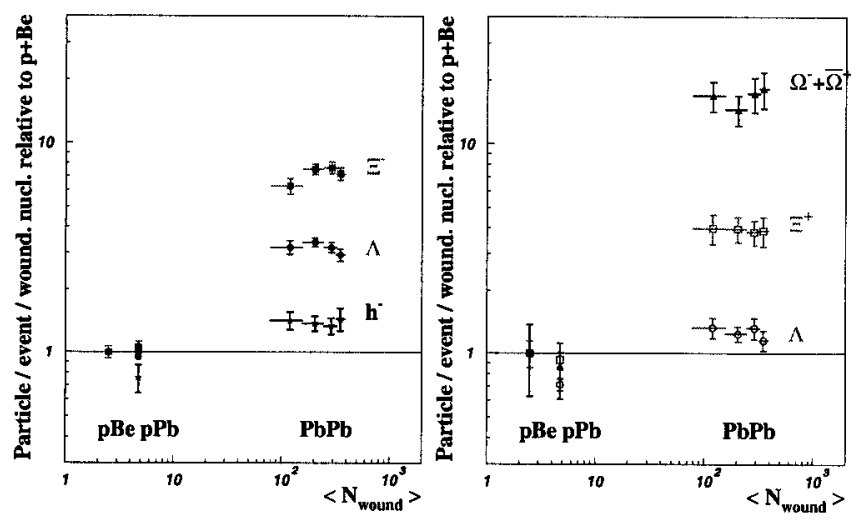

Figure 1. WA97 Particle yields relative to p-Be, divided by the number of wounded nucleon $N_{\text {wound }}$ as a function of $N_{\text {wound }}$, assumed as definition of the number of participants.

The NA57 experiment studies the production of strange and multi-strange baryons and antibaryons in ultrarelativistic nucleus-nucleus collisions at the SPS.

The main aim of NA57 is to investigate the behaviour of the enhancement of the production of particles with strangeness $|s|=1,2,3$ in nucleus-nucleus collisions at the variation of the energy and of the centrality of the collision defined e.g. as the number of participant nucleons.

We shall recall the main features of the experimental set-up, and we shall illustrate the collected data samples and the status of their analysis.

\section{Introduction}

The NA57 experiment [1] studies the production of strange and multi-strange baryons and antibaryons in ultrarelativistic nucleus-nucleus collisions at the SPS. The results from the previous experiment WA97 [2] show that the strange hyperons abundances increase faster than the number of participants in the collision when going from proton-nucleus to nucleus-nucleus interactions. The strangeness enhancement shows a hierarchical pattern, being stronger for the hyperons of higher strangeness content, as shown in fig. 1. This effect was predicted [3] as a signature of a phase transition from hadronic matter to a plasma of deconfined quarks and gluons.

The main aim of NA57 is to investigate the behaviour of the enhancement of the production of particles with strangeness $|s|=1,2,3$ in nucleus-nucleus collisions at the variation of the energy and of the centrality of the collision defined e.g. as the number of participant nucleons.

NA57 has taken data with $\mathrm{Pb}$ beams of $158 A \mathrm{GeV} / \mathrm{c}$ and $40 A \mathrm{GeV} / \mathrm{c}$ momentum. The latter corresponds to a centre-of-mass energy $(8.8 \mathrm{GeV} / \mathrm{c}$ per nucleon) intermediate between that achieved at the AGS (4.7 GeV/c per nucleon) and the top energy available 
at the SPS $(17.3 \mathrm{GeV} / \mathrm{c}$ per nucleon for a $\mathrm{Pb}$ beam). A sample of p-Be data at $40 \mathrm{GeV} / \mathrm{c}$ has been also collected for reference.

\section{The experimental apparatus}

The main experimental challenge is to handle the large density of primary tracks produced in central lead-lead interactions. To reconstruct $\Lambda, \Xi, \Omega$ and $K$ decays in such an environment, we employ a high granularity telescope of silicon pixel planes.

The apparatus, shown schematically in fig. 2, is placed inside the GOLIATH magnet which provides a maximum field of $1.4 \mathrm{~T}$. The main features of the apparatus are:

- a telescope made of 13 silicon pixel detector planes, for a total of about $1.1 \times 10^{6}$ channels; 7 planes use the Omega2 [4] front end chip with a pixel size of $75 \times 500$ $\mu \mathrm{m}^{2}, 6$ planes use the Omega3 [5] front end chip, with a cell size of $50 \times 500 \mu \mathrm{m}^{2}$. The silicon pixel technique was succesfully pioneered by WA97 in collaboration with RD19 [6]. The mechanical support of the telescope allows to change its position with respect to the target. The telescope is placed above the beam line and it is inclined in such a way that the lower edges of the detectors lay on a line pointing to the target.

Hyperons and $\mathrm{K}$ mesons are identified via the following decay channels: $\Lambda \rightarrow p+\pi^{-}$ $\Xi^{-} \rightarrow \Lambda+\pi^{-}, \Omega^{-} \rightarrow \Lambda+K^{-}, K^{0} \rightarrow \pi^{+}+\pi^{-}$and $K^{ \pm} \rightarrow \pi^{+}+\pi^{-}+\pi^{ \pm}$. As an example, fig 3 shows an $\Omega^{-}$decay topology with all the decays tracks in the telescope acceptance. In both the $158 \mathrm{AGeV} / \mathrm{c}$ and $40 \mathrm{AGeV} / \mathrm{c}$ run, the telescope was set-up so as to have maximum acceptance at mid rapidity.

- an array of 6 scintillator petals, placed $10 \mathrm{~cm}$ downstream of the target, covering the pseudorapidity region $1<\eta<2$, used to trigger on the centrality of the collision.

- two planes of multiplicity silicon detectors (MSD) sampling the charged multiplicity at mid-rapidity, used in the determination of the centrality of the nucleus-nucleus collision.

\section{Multiplicity measurement}

The centrality of the collision is measured by sampling the charged multiplicity at central rapidity, with two stations of Multiplicity Strip Detectors (MSD).

The NA57 and WA97 experiments have similar layouts, and in particular they employ the same multiplicity detectors (MSD, see figure 4). In NA57 additional efforts were made to reduce the empty target contamination in the triggered sample, in order to extend the covered centrality range towards more peripheral events. The two MSD planes were placed respectively $19.8 \mathrm{~cm}$ and $54.5 \mathrm{~cm}$ downstream of the target for the $158 A \mathrm{GeV} / \mathrm{c}$ run. In the $40 \mathrm{~A} \mathrm{GeV} / \mathrm{c}$ configuration the planes were positioned $20.4 \mathrm{~cm}$ and $38 \mathrm{~cm}$ from the target .

With this geometry, the first and the second plane cover approximately the pseudorapidity regions $2<\eta<3$ and $3<\eta<4$ for the $158 \mathrm{~A} \mathrm{GeV} / \mathrm{c}$ run, and $2<\eta<3$ and 


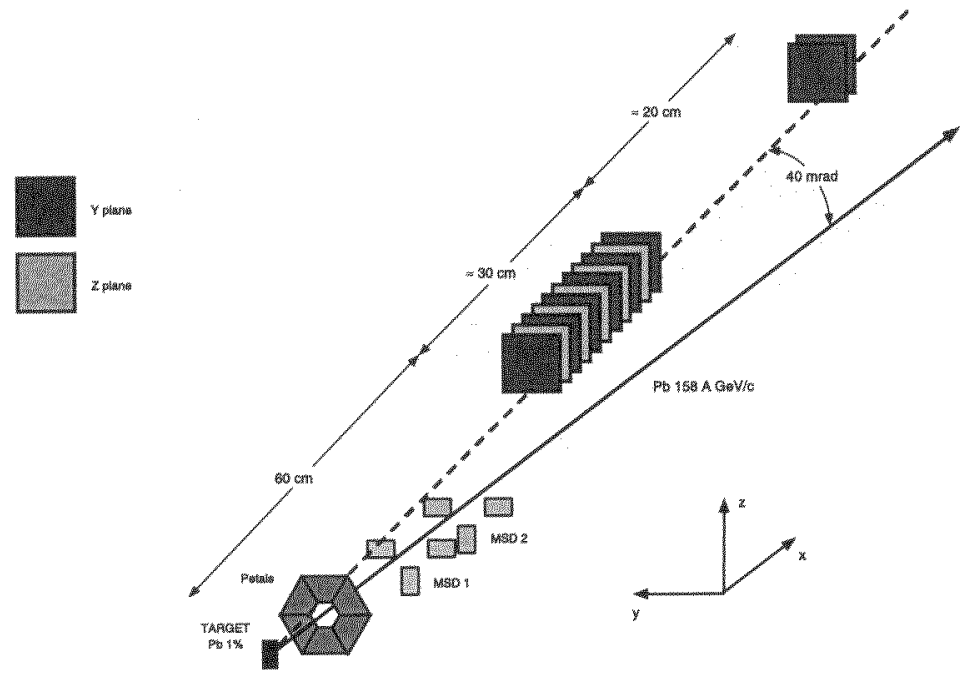

Figure 2. A 3D view of the NA57 apparatus.

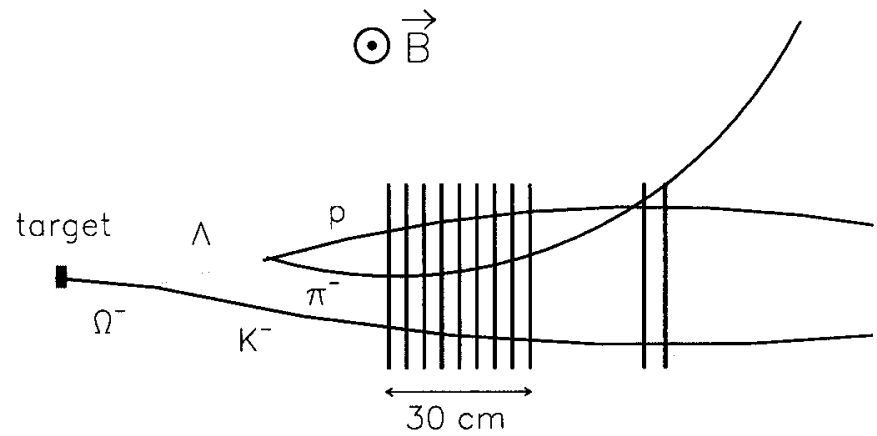

Figure 3. An example of a $\Omega^{-}$decay topology, with all the decays tracks in the telescope acceptance. 


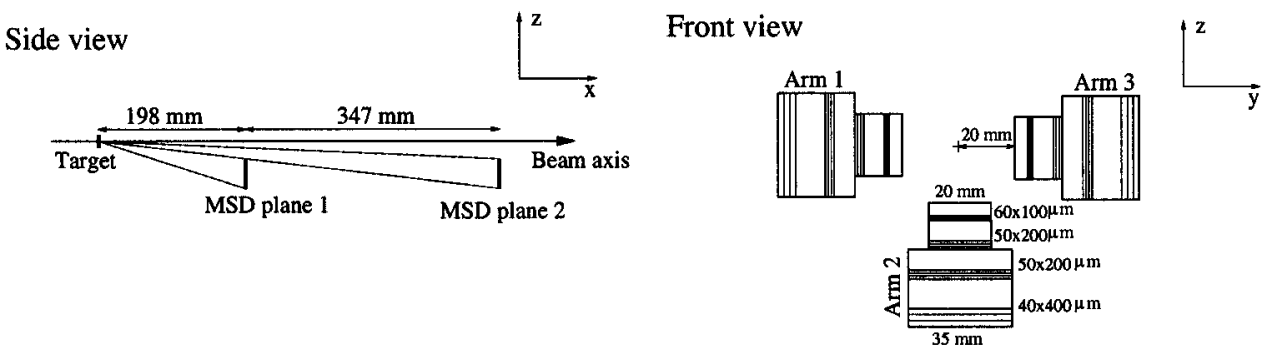

Figure 4. Layout of the micro-strip multiplicity detectors (158 $A \mathrm{GeV} / \mathrm{c}$ run). Only the lower arm is shown on the side view.
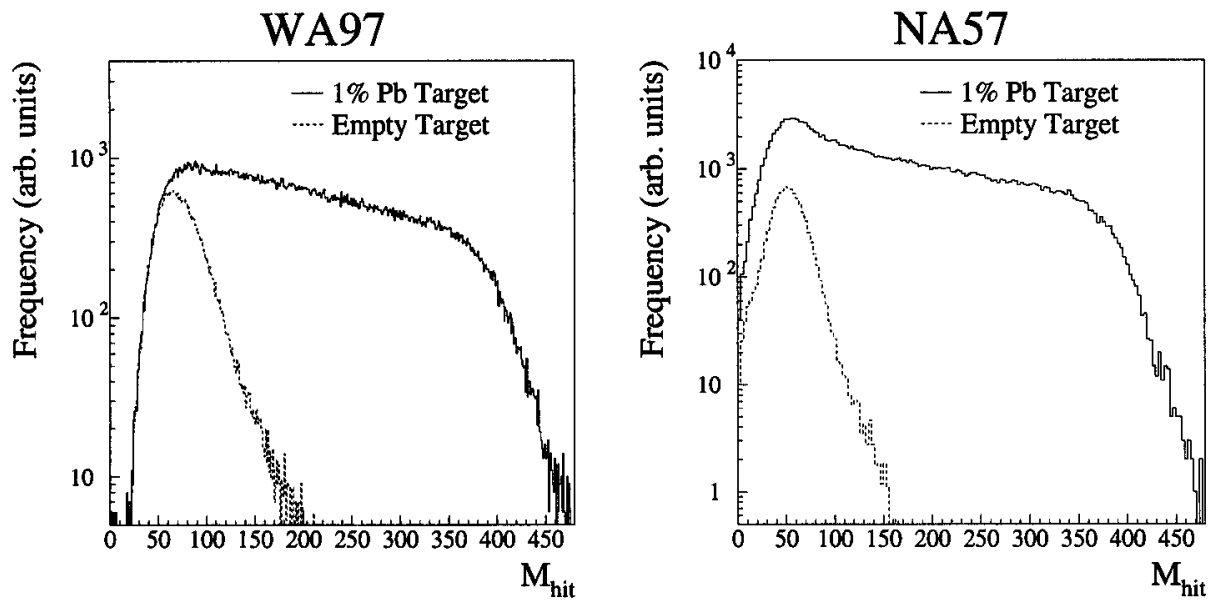

Figure 5. Hit multiplicity distribution of the triggered events with a $1 \% \mathrm{~Pb}+\mathrm{Pb}$ interaction length target (solid histogram) and of the empty target sample (dashed histogram). 


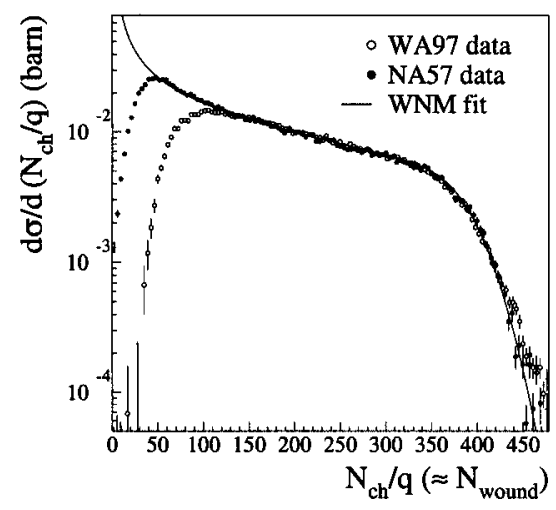

Figure 6. Fit of the multiplicity distributions with the Wounded Nucleon Model for the WA97 and NA57 experiments.

$2.4<\eta<3.7$ for the $40 \mathrm{~A} \mathrm{GeV} / \mathrm{c}$ run. The azimuthal acceptance is about $30 \%$ for both planes.

The distributions of the total hit multiplicity in the two detector planes for the WA97 and NA57 experiments $(158 \mathrm{~A} \mathrm{GeV} / \mathrm{c})$ are shown in figure 5. The drop at low multiplicities is the effect of the scintillator petals centrality trigger suppressing low multiplicity events.

The contamination of the triggered sample resulting from interactions in air or other materials along the beam line (empty target contamination) was evaluated using data collected without the target and then subtracted. It was $\simeq 17 \%$ of the triggered events for WA97 and $\simeq 6 \%$ for NA57. Figure 5 shows the multiplicity distributions for the $1 \% \mathrm{~Pb}+\mathrm{Pb}$ interaction length target and for the empty target runs. Details on the multiplicity recostruction procedure can be found in [7].

The multiplicity distribution can be described in the framework of the wounded nucleon model (WNM) [8]. In this model, it is assumed that the average charged particle multiplicity is proportional to the number of wounded nucleons, computed from the Glauber model [9]:

$\left\langle N_{c h}\right\rangle=q N_{\text {wound }}$

where $q$ is a proportionality constant. The only physical inputs to the model are the density distribution of the nucleons inside the nucleus and the nucleon-nucleon cross section.

The corrected WA97 and NA57 charged particle multiplicity distributions in the range $2<\eta<4$ are shown in fig. 6 , together with the fitted curve. In the abscissa the number of charged particles is divided by the fitted scale factor $q$; the resulting variable, which - 


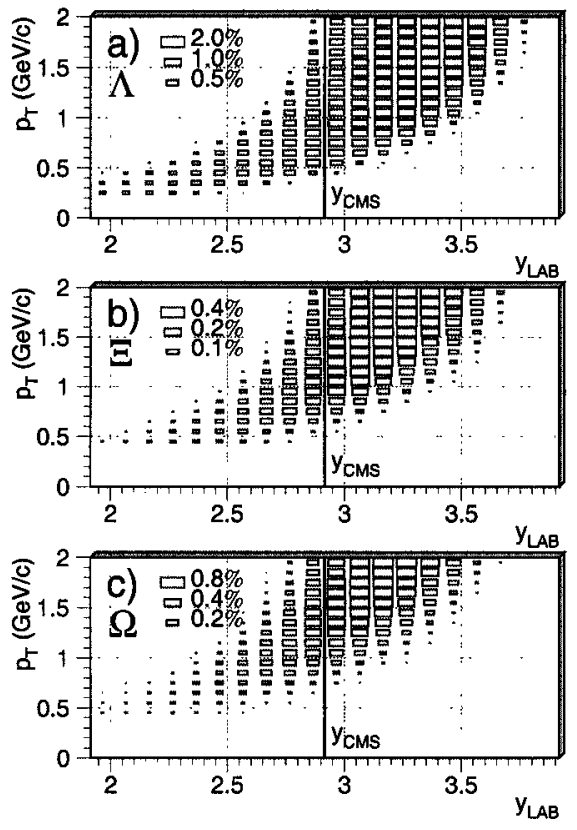

Figure 7. NA57 acceptances table for strange particles $(158 \mathrm{~A} \mathrm{GeV} / \mathrm{c})$.

apart for the experimental and physical smearing - is close to $N_{\text {wound }}$, is used to compare directly the centrality distributions from the two experiments.

As can be seen from fig. 6, NA57 extend the centrality range down to events with $N_{\text {wound }} \simeq 50$. The fitted trigger cross section for the WA97 experiment is compatible with the estimated experimental value of about $40 \%$ of the total $\mathrm{Pb}+\mathrm{Pb}$ inelastic cross section. In the NA57 experiment the trigger cross section was measured with a $3 \%$ precision and its value, $\sigma_{\text {trig }}^{\exp }=(4.29 \pm 0.12)$ barn, corresponding to about $60 \%$ of the total nuclear inelastic cross section, is in agreement with the value obtained from the WNM fit.

\section{The data sample}

\section{1. $\mathrm{Pb}-\mathrm{Pb}$ at $158 A \mathrm{GeV} / \mathrm{c}$}

In 1998 we collected about $230 \mathrm{M}$ events, with the set-up shown in fig. 2. The telescope was inclined at $40 \mathrm{mrad}$ and the first plane was placed $60 \mathrm{~cm}$ downstream of the target.

The acceptance tables for $\Lambda, \Xi$, and $\Omega$, reported in figure 7 , are similar to the corresponding ones in WA97. The full data sample has been processed through the track finding and reconstruction program ORHION. A partial statistics $\Xi+\bar{\Xi}$ invariant mass distribution is shown in fig. 8 as an example. 


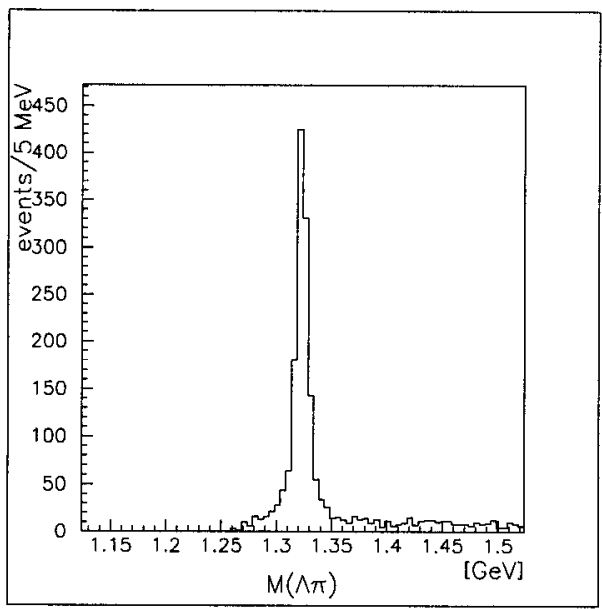

Figure 8. Invariant mass of $\Lambda-\pi$ (+c.c.) pairs from a partial sample collected at $158 \mathrm{~A}$ $\mathrm{GeV} / \mathrm{c}$.

\section{2. $\mathrm{Pb}-\mathrm{Pb}$ and $\mathrm{p}-\mathrm{Be}$ at $40 \mathrm{AGeV} / \mathrm{c}$}

In 1999 we collected a sample of $260 \mathrm{M}$ of $\mathrm{Pb}-\mathrm{Pb}$ interactions at $40 \mathrm{~A} \mathrm{GeV} / \mathrm{c}$. In order to have maximum acceptance at mid rapidity the telescope was inclined at $72 \mathrm{mrad}$. The first plane was positioned $40 \mathrm{~cm}$ downstream of the target. The acceptance tables for this configuration are shown in fig. 9. The alignment and the calibration of the detector for this set of data are going on, and the multiplicity analysis is in progress.

In addition, a sample of $60 \mathrm{M}$ p-Be interactions at $40 \mathrm{GeV} / \mathrm{c}$ was also collected with the same set-up. The beam was in this case a mixture of pions and protons (about $40 \%$ of the total), which were identified by two Čerenkov counters.

\section{Conclusions}

The NA57 experiment can play a unique role in the investigation of the onset of a phase transition from hadronic matter to Quark Gluon Plasma by addressing the two main questions arising from WA97 results, namely: i) how strange particle yields behave at lower values of the number of nucleons participating to the collision and ii) how this behaviour depends on the centre-of-mass energy of the collision.

The WNM analysis of the multiplicity distribution shows that we collected statistics for a centrality range extended down to $N_{\text {wound }} \simeq 50$.

The physical analysis of the data samples at $158 A \mathrm{GeV} / \mathrm{c}$ and at $40 A \mathrm{GeV} / \mathrm{c}$ is in progress. 

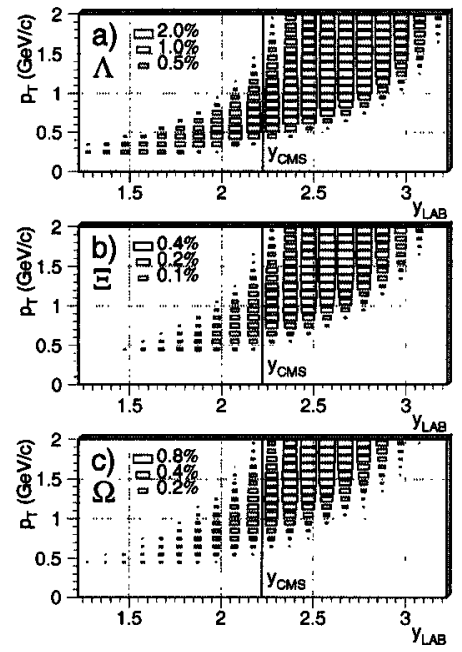

Figure 9. NA57 acceptance table for strange particles $(40 A \mathrm{GeV} / \mathrm{c})$.

\section{REFERENCES}

1. R. Caliandro et al., NA57 proposal, CERN/SPSLC 96-40 SPSLC/P300.

2. WA97 Collaboration: E. Andersen et al., Phys. Lett. B433 (1998) 209.

WA97 Collaboration: E. Andersen et al., Phys. Lett. B449 (1999) 401.

3. J. Rafelski and B. Müller, Phys. Rev. Lett. 48, (1982) 1066.

4. M. Campbell et al., Nucl. Instr. and Meth. A290 (1990) 149.

5. E.H.M. Heijne et al., Nucl. Instr. and Meth. A383 (1996) 55.

6. RD19 Collaboration: E.H.M. Heijne et al., Nucl. Instr. Meth. A349 (1994) 138

7. F. Antinori et al., CERN preprint CERN-EP-2000-002 (to be published in Eur. Phys. J. C).

8. A. Białas, M. Bleszyński and W. Czyż, Nucl. Phys. B111 (1976) 461.

9. C.Y. Wong, Introduction to High-Energy Heavy-Ion Collisions (World Scientific Publishing, Singapore 1994) 251-264. 